\title{
The Study of the effects of Free Carriers Trap on the Recombination Mechanism through the Localized States
}

\author{
Tamire Legesse Fole ${ }^{1 *} \quad$ Megersa Wodajo Shura ${ }^{2} \quad$ Tadese Debele Gebre $^{1}$ \\ 1.Department of Physics, Debark University, Debark, Ethiopia \\ 2.Department of Physics, Adama science and Technology University, Adama, Ethiopia
}

\begin{abstract}
In this study, the effects of free carriers trap on the recombination mechanism through the localized states in gallium antimonide $(\mathrm{GaSb})$ is investigated through the simulation of excess carrier traps corresponding to the nonradiative recombination mechanism. Fist, the trapping effects (the ratio of the concentration of trapped carriers to the corresponding concentration of free carriers) is described as a function localized state energy at room and different temperatures inside the sample. Then, the radiative, Auger and Shockley-Read-Hall recombination mechanisms are compute using the relations describing the lifetimes of the excess majority and minority carriers in the band gap of semiconductor(GaSb). Finally, the temperature dependence of the radiative, Auger and Shockley-Read-Hall excess carrier lifetimes in the localized regions are investigated by considering the variation of localized energy positions. Since trap is indirectly related to the temperature, the description of the trapping effect as functions of localized state energies is found to be the important. The analysis of the results also shows that, the under localized regions are divided in to five based on the interaction of localized state energy with the conduction band energy or the valance band energy and decreases with temperature. The trap in shallow levels (donor and acceptor regions) tends to zero and deep levels are dominated at high temperature. The Auger lifetime is dominated than the other (radiative and Shockley-Read-Hall) lifetimes for the sample semiconductor $(\mathrm{GaSb})$. The temperature affects both majority and minority carriers trap and their lifetimes in the three recombination mechanisms as functions of the localized state energies and the Auger lifetime is dominated in the semiconductor gallium antimonide sample.
\end{abstract}

Keywords: Free Carriers Trap, localized States, Shockley-Read-Hall, Auger band-to-band recombination

DOI: $10.7176 / \mathrm{JNSR} / 9-5-03$

Publication date:March $31^{\text {st }} 2019$

\section{Introduction}

Semiconductors are a group of materials having electrical conductivities between metals and insulators[1].The conductivity of these materials can be varied over orders of magnitude by changes in temperature, optical excitation, and impurity content. The column IV semiconductors, silicon and germanium are called elemental semiconductors because they are composed of single species of atoms[2]. Every solid has its own characteristic energy band structure. This variation in band structure is responsible for the wide range of electrical characteristics observed in various materials. Semiconductor materials at $0 \mathrm{~K}$ have basically the same structure as insulators. In a semiconductor, the energy difference between the top of valance band $\left(E_{V}\right)$ and the bottom of conduction $\left(E_{C}\right)$ is defined as the band-gap energy $\left(E_{g}\right)$. The band gap energy $E_{g}=E_{C}-E_{V}$ is perhaps the most important in semiconductor physics. A contraction of the crystal lattice with decreasing temperature usually leads to a strengthening of the interatomic bonds and an associated increase in the band gap energy. The first empirical relation for the band gap shift with temperature was developed by Varshni et al[3, 4]

$$
E_{g}(T)=E_{g}(0)-\frac{\alpha T^{2}}{T+\beta},
$$

where $\alpha=3.78 \mathrm{e} / \mathrm{K}$ and $\beta=94 \mathrm{~K}$ are constants chosen to obtain the best fit to experimental data and $E_{g}(0)=0.813 \mathrm{eV}$ is the limiting value of the band at zero Kelvin for GaSb.

In metals, the bands either overlap or are only partially filled. Thus in metals, high electrical conductivity is due to the fact that electrons and empty energy states are intermixed within bands so that electrons can move freely under the influence of an electric field [5].

A. Mitonneau and A. Mircea, et al studied electron and holes capture cross-sections at deep centers in gallium arsenide by using the method, Deep Level Transient Spectroscopy (D.L.T.S.), has led to a better knowledge of the many different deep levels which one can find in the forbidden band gap of the semiconductors [6].

In this paper, we would have demonstrated the traps and the lifetime properties through the energy band gap (localized state) by using the method, Microsoft excel and Origin. And a thorough discussion on the topic of the study of the effects of free carriers trap on the recombination mechanism through the localized states will be presented. Un-doped GaSb is used as the primary sample throughout this study. The competition between different localized states in the recombination mechanism and trapping of free carriers is studied. The effects temperature 
and the energy level of the localized states on the recombination and trapping of free carriers is also studied. The results of this research will broaden the knowledge of scientific community on optoelectronics devices by presenting the theoretical description of free carrier trapping effects in the small and direct band-gap semiconductor $(\mathrm{GaSb})$. Moreover, the result obtained from this paper will be used as an input for other researchers.

\section{Materials and Methodology}

The effects of trapping on the SRH excess carrier lifetime where studied by substituting the density of the trapped carriers in the relations describing the recombination mechanisms. The effect of the temperature on the density of the carriers trapped at different localized states also studied. The radiative lifetime $\left(\tau_{R}\right)$,

Auger lifetime $\left(\tau_{A}\right)$ and SRH lifetime $\left(\tau_{S R H}\right)$ for excess carriers in p-type GaSb material are determined as functions of localized at different temperature state. We calculated and plotted all graphs by Microsoft excel and origin using the standard formulas.

\section{Results and discussion}

Under this subtopic we would deals with the analysis, discussion and the results of this paper. First, the trapping effects at room and at various temperatures in different regions of semiconductor $(\mathrm{GaSb})$ were analyzed. Next, the computation of radiative, Auger and SRH recombination mechanisms through their recombination lifetimes described. Finally, the radiative-lifetime $\left(\tau_{R}\right)$, Auger lifetime $\left(\tau_{A}\right)$ and SRH lifetime $\left(\tau_{S R H}\right)$ for excess carriers in p-type GaSb materials were analyzed as functions of localized state energy.

\subsection{The temperature dependence of trapping effects}

Figure 5(a) and (b) illustrates the trap effects $\boldsymbol{I}_{\boldsymbol{n}}$ and $\boldsymbol{I}_{\boldsymbol{p}}$ as functions of energy position of the trap level for undoped p-type gallium antimonide (GaSb), (a) at $300 \mathrm{~K}$ and (b) at different temperatures. The graphs are drawn using the following Equations.

and

$$
I_{n}=\frac{\left(\tau_{p 0} p_{0 T}-\tau_{n 0} n_{0 T}\right)}{\tau_{n 0}\left(p_{0}+p_{1}+n_{0 T}\right)+\tau_{p 0}\left(n_{0}+n_{1}\right)}
$$

$$
I_{p}=\frac{\left(\tau_{n 0} n_{0 T}-\tau_{p 0} p_{0 T}\right)}{\tau_{n 0}\left(p_{0}+p_{1}\right)+\tau_{p 0}\left(n_{0}+n_{1}+p_{0 T}\right)} .
$$

for a continuous distribution of impurity states with uniform trap level concentrations equal to the carrier concentration throughout the bulk. This helps to understand the interplay between different localized states and the free carrier concentrations at different temperatures[7]. Figure 5 (a) shows both the effects of the majority and minority carrier traps at a relatively room temperature $(300 \mathrm{~K})$. These result analyzed by fixing the total density of localized state value at $N_{T}=10^{17} \mathrm{~cm}^{-3}$, doping level at $\boldsymbol{N}_{\boldsymbol{a}}=\mathbf{1 0}^{\mathbf{1 8}} \mathrm{cm}^{-3}$ value and injection level $\boldsymbol{G}_{\mathbf{0}}=\mathbf{0}$ agreed within[3].
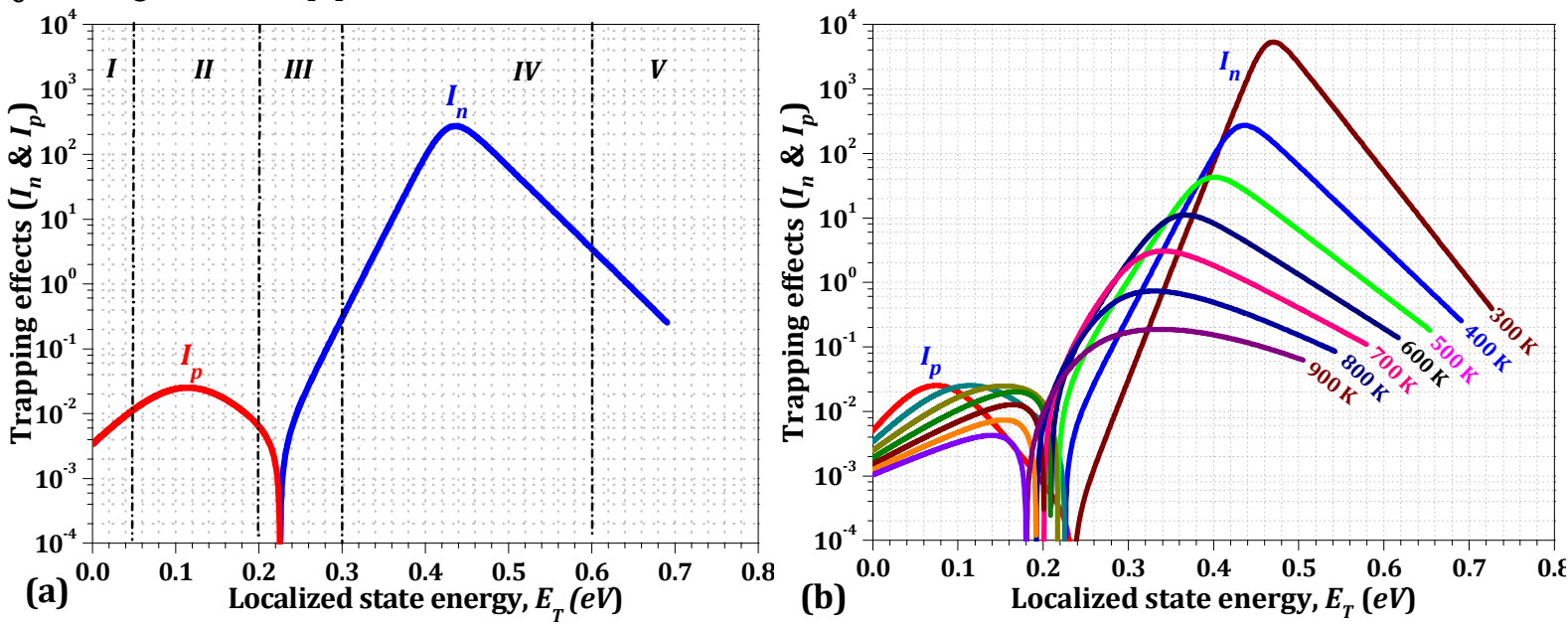

Figure 5:The free carriers trapping effect as a function of energy of the trap level for (a) room and (b) different temperatures.

In general, free carrier traps are relatively inactive at high temperatures compared to low temperatures. Only deep levels can trap free carriers at higher temperatures as shown in Figure 5(a). The minority carrier traps farther away above the intrinsic level become more effective with decreasing temperature as shown in Figure 5(b). Depending on the interactions of localized states with the conduction or valence bands, the energy level dependence plot of the trap in Figure 5 (a) can be divided into five regions labeled I through $\mathrm{V}$. 
Region I: This region including all the energy levels very close to the valence band edge (from valance band energy to $0.05 \mathrm{eV}$ ). The energy value of this region is approximately equal or less than to thermal energy $k_{B} T$. Most of the impurities are in the $n_{T}$ state (When we compare the density of states $n_{0 T}$ with $p_{0 T}$, the $n_{0 T}$ state is much greater than $p_{0 T}$ ) and the hole emission rate is very high. But,

$$
n_{0 T}=f_{n} N_{T}=\frac{N_{T}}{1+\exp \left(\frac{E_{T}-E_{F}}{k_{B} T}\right)}, \quad p_{0 T}=N_{T}-n_{0 T} .
$$

Hence the effects of the traps of a localized center in both Eqns. (3.1) and (3.2), become negligibly small in this region. The localized centers hence act as acceptor levels. Therefore, the region is in $n_{0 T}$ state and we can call this region acceptor region[8].

Region II: This region includes all the energy levels far above the valence band edge and far below the intrinsic Fermi level (from the localized energy level $0.05 \mathrm{eV}$ to $0.2 \mathrm{eV}$ ). The energy value of this region is much greater than thermal energy $\mathrm{k}_{\mathrm{B}}$ Tor $\left(E_{T} \gg k_{B} T\right)$. In this region the capture cross section of hole much greater than capture cross section of electrons $\left(\sigma_{p} \gg \sigma_{n}\right)$. Where:-

$$
\sigma_{p}=\sigma_{p \infty} \exp \left(-\frac{E_{T}-E_{V}}{k_{B} T}\right), \quad \sigma_{n}=\sigma_{n \infty} \exp \left(-\frac{E_{c}-E_{T}}{k_{B} T}\right),
$$

Where:- the constant $\sigma_{p \infty}=10^{16} \mathrm{~cm}^{2}$ and $\sigma_{n \infty}=10^{15} \mathrm{~cm}^{2}$.

When we compare the density of states $\left(n_{0 T}\right.$ with $\left.p_{0 T}\right), \mathrm{n}_{0 T}$ is greater than $p_{0 T}\left(n_{0 T}>p_{0 T}\right)$. Since the capture cross section represents the probability of traps can be capturing the free carries, the probability of holes capture is greater than the probability of electron capture and emission rate of holes are decreased in this region. In this region specially from the energy level $0.08 \mathrm{eV}$ to $0.15 \mathrm{eV}$, hole trapping has maximum values and thus the hole trapping effect becomes dominant as shown in Figure 5a. Therefore, we can call this region holes trapping region.

Region III: This region consists all the energy trap level very closer to the intrinsic Fermi level in both sides, where the localized levels are partially ionized. According to this sample the range of the region includes trap center from the energy level $0.2 \mathrm{eV}$ to $0.3 \mathrm{eV}$. In this region almost the capture cross section of holes and capture cross section of electrons are equal $\left(\sigma_{p}=\sigma_{n}\right)$. So that the effects of free charge carrier traps are negligibly small as shown in Figure 5 above and the probability of holes capture is equal to the probability of electron capture. The $\mathrm{SRH}$ recombination becomes dominant in this region i.e. recombination takes place. Therefore, we have called this region recombination region.

Region IV: This region including all the energy levels that are very close to the intrinsic Fermi level and most of the regions far below the conduction band energy (from the energy level $0.3 \mathrm{eV}$ to $0.6 \mathrm{eV}$ ). In this region specially, at the energy level equal to $0.42 \mathrm{eV}$, the electron trapping has maximum values. Most localized states are in the $p_{T}$ state and the electron capture rate is very high and in this region the capture cross -section of electrons much greater than capture cross section of holes $\left(\sigma_{n} \gg \sigma_{p}\right)$. When we compare the density of states $\left(p_{0 T}\right.$ with $\left.n_{0 T}\right)$, $n_{0 T}$ is greater than $p_{0 T}\left(n_{0 T}>p_{0 T}\right)$. So, the probability of capture electron is greater than the probability of hole capture or emission rate of electron are high in this region[3]. The electron trap effect becomes the most dominant as shown in Figure 5a. The levels of the effective electron traps at room temperature is in complete agreement with the results reported by Habegger et al in p-type GaSb samples. Therefore, we have called this region electron trap region.

Region $\boldsymbol{V}$ : This region including all the localized energy levels (from $0.6 \mathrm{eV}$ to conduction band energy) which is closer to the conduction band edge. The energy value of this region is approximately equal or less than to thermal energy $k_{B} T$. Most of the impurities are in the $p_{T}$ state (When we compare the density of states $n_{0 T}$ with $p_{0 T}$, the $p_{0 T}$ state is much greater than $n_{0 T}$ ) and the electron emission rate is very high. Hence, the effects of the traps of a localized center in both Equations:-

and

$$
I_{n}=\frac{\left(\tau_{p 0} p_{0 T}-\tau_{n 0} n_{0 T}\right)}{\tau_{n 0}\left(p_{0}+p_{1}+n_{0 T}\right)+\tau_{p 0}\left(n_{0}+n_{1}\right)}
$$

$$
I_{p}=\frac{\left(\tau_{n 0} n_{0 T}-\tau_{p 0} p_{0 T}\right)}{\tau_{n 0}\left(p_{0}+p_{1}\right)+\tau_{p 0}\left(n_{0}+n_{1}+p_{0 T}\right)} .
$$

, become negligibly small in this region. The localized centers hence act as donor levels. Therefore, the region is in $p_{0 T}$ state and we can call this region donor region.

Generally, we can analyze that the localized energy found between the value from valance band edge to $0.05 \mathrm{eV}$ has no carriers trap, the localized energy levels found between $0.05 \mathrm{eV}$ to $0.2 \mathrm{eV}$, has high hole traps and $0.3 \mathrm{eV}$ to $0.6 \mathrm{eV}$ has high electron trap and the value from conduction band edge to $0.6 \mathrm{eV}$ has no carriers traps for 
semiconductor material (GaSb) samples. And as the temperature is increases, the trap effect is decreasing.

\subsection{The computation between the radiative, Auger and SRH recombination lifetimes}

Figure 6illustrates the radiative, Auger and SRH recombination mechanisms through their recombination lifetimes at room temperature as a function of localized state energy in gallium antimonide samples (a) excess electrons lifetime and (b) excess hole lifetime. These result analyzed by fixing the total density of localized state value at $N_{T}=10^{17} \mathrm{~cm}^{-3}$, doping level at $N_{a}=10^{18} \mathrm{~cm}^{-3}$ value and injection level $\mathrm{G}_{0} \approx 0$ (low injection level).

From Figure 6(a) the minority carrier SRH lifetime (blue line) is the largest one and it depends on the energy of localized states i.e. there is no recombination and trapping of carriers for localized levels near to the valence and conduction band edges (acceptor and donor region). Also, this tells us the SRH excess recombination rate $U_{S R H}$ is much smaller than excess recombination rate of radiative $\left(U_{R}\right)$ and Auger $\left(U_{A}\right)$, since the carrier lifetimes calculated by the ratio of carrier concentration $(\delta n)$ to recombination rate $\left(U_{S R H}\right)$ as shown in equation given below:-

$$
\begin{gathered}
\tau_{n S R H}^{\prime}=\frac{\delta n}{U^{\prime}{ }_{S R H}}=\frac{\tau_{n 0}\left(p_{0}+p_{1}+\delta p\right)+\tau_{p 0}\left(n_{0}+n_{1}+\delta n\right)}{\left(p_{0}+\left(n_{0}+\delta n\right) \delta p / \delta n\right.} . \\
\text { Or } \quad \\
\tau_{n S R H}^{\prime}=\frac{\left.\tau_{n 0}\left(p_{0}+p_{1}+\delta p\right)\left(1+I_{n}\right)+\tau_{p 0}\left(n_{0}+n_{1}\right)\left(1+I_{n}\right)+\delta p\right)}{\left(p_{0}+n_{0}\left(1+I_{n}\right)+\delta p\right)\left(1+I_{n}\right)} .
\end{gathered}
$$

Again, from Figure 6 (a) shows the minority carrier (electron) radiative lifetime (black line) is the larger one and the Auger lifetime (red line) the smallest and both are independent of the energy of localized states.
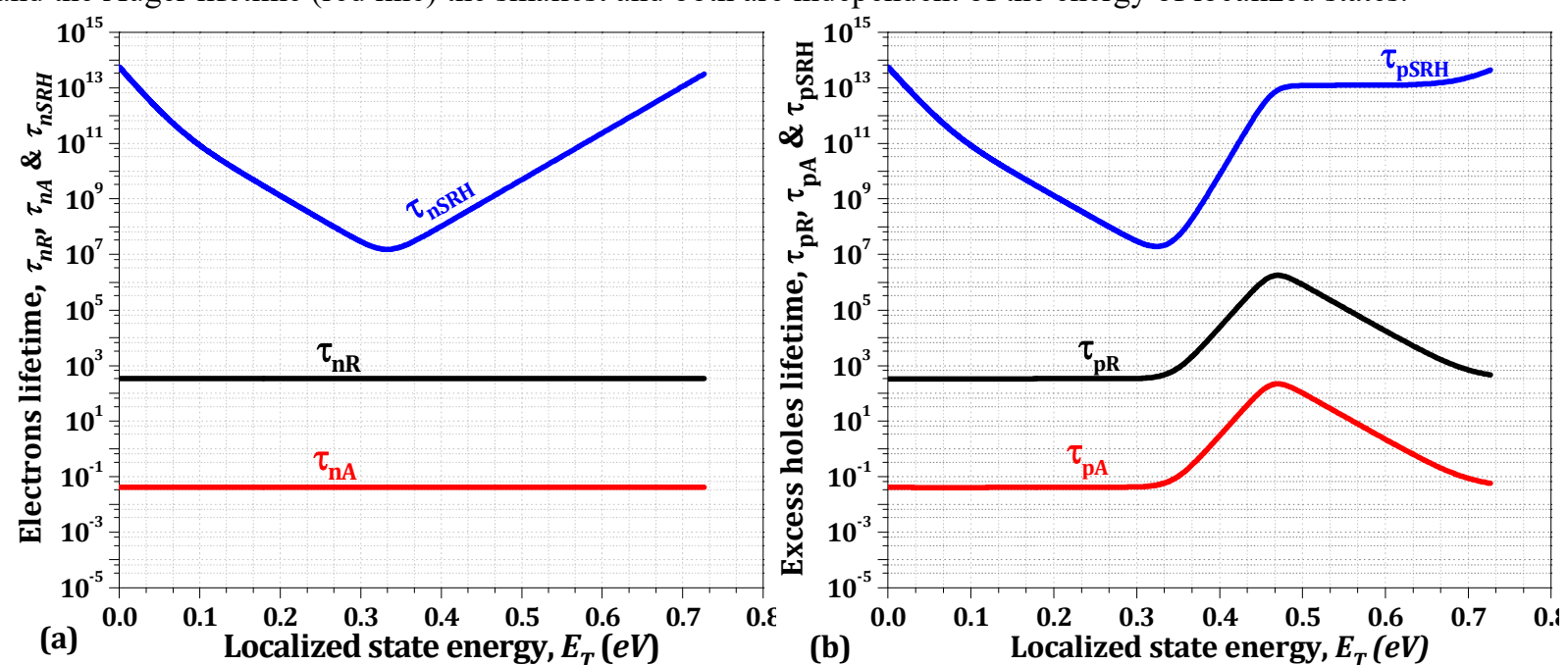

Figure 6: The computation between radiative, Auger and SRH recombination mechanisms as function of localized state energy for (a) the excess electron (b) the excess hole lifetimes.

The minority carrier radiative lifetime is larger and it is constant over the whole region which is independent of the localized state energy. This tells us radiative excess minority carrier lifetime under the localized region or forbidden gap is remain constant for all localized energy level as shown in Figure 6(a). The minority carrier Auger lifetime is small and other explanation is similar to radiative lifetime (see Figure 6 (a)). Both radiative and Auger excess carrier lifetime in the localized region (valance to conduction band) in the semiconductor material (GaSb) remain constant at different localized energy levels[10]. The Auger excess minority carrier lifetime in the semiconductor material (GaSb) dominate at different localized energy levels as shown Figure 6a.

From Figure 6(b) majority carrier SRH lifetime is the largest, radiative lifetime larger, Auger lifetime small and all are the functions of the localized state energies. This shows us again the Auger excess majority carrier lifetime in the semiconductor material $(\mathrm{GaSb})$ dominate at different localized energy levels as shown in Figure 6 (b). Therefore, the Auger excess carriers' lifetime dominated compared to the radiative and SRH excess carriers' lifetimes.

\subsection{The temperature dependence of Radiative and Auger lifetimes}

Figure 7:illustrates the radiative and Auger lifetimes at different temperatures as a function of localized state energy for (a) radiative excess electrons, (b) radiative excess holes, (c)Auger excess electrons and (d) Auger excess holes. The graphs are drawn by using the ratio of Equation (3.9) to (3.10) for radiative

$$
U_{R}=C_{R} \delta n\left(\delta n+p_{o}+n_{o}\right) \text {. }
$$


Where:-

$$
\delta n=\frac{\delta N}{\left(1+I_{n}\right)}, \quad \delta p=\frac{\delta N}{\left(1+I_{p}\right)},
$$

$I_{n}$ and $I_{p}$ are electron and hole trap respectively.

And Equation (3.11)for Auger.

Where:-

$$
\tau_{A}=\frac{\tau_{A 0}}{1+\tau_{A 0}\left(k_{A} \delta n+C_{A} \delta n^{2}\right)^{\prime}}
$$

$$
\begin{gathered}
\tau_{A 0}=\frac{1}{C_{p a}\left(p_{0}^{2}+2 n_{i}^{2}\right)+C_{n a}\left(n_{0}^{2}+2 n_{i}^{2}\right)}, \quad C_{A}=C_{p a}+C_{n a} . \\
k_{A}=C_{p a}\left(2 p_{0}+n_{0}\right)+C_{n a}\left(2 n_{0}+p_{0}\right) .
\end{gathered}
$$

As describe above Figure 6, the carrier lifetime can be calculated as the carrier concentration divided by recombination of carriers. Therefore, based on this calculation the radiative electrons lifetime remains constant over the whole regions as function of localized state energies and increases with temperatures below $800 \mathrm{~K}$ but no changes above (for $900 \mathrm{~K} \& 1000 \mathrm{~K}$ )[11]. The radiative hole lifetime varies entire the whole regions and reaches a maximum value in electron trap region for low temperature $(300 \mathrm{~K})$ for this sample and increases with temperatures in the acceptor, donor and hole trap regions. But decreases with temperatures in electron trap region. The Auger excess electrons lifetimes is constant over the whole regions and increases for room temperature to 600 $\mathrm{K}$ then decreases above [8]. The Auger excess electrons lifetimes shows similar distribution to the radiative hole lifetime varies entire the whole regions.

Generally, the radiative and Auger lifetimes in general increase then decrease with temperature as shown in Figure 7. Both radiative and Auger excess minority carrier lifetime in the localized region (valance to conduction band) in the semiconductor material (GaSb) remain constant and varies for excess majority carrier lifetime at different localized energy levels[11].
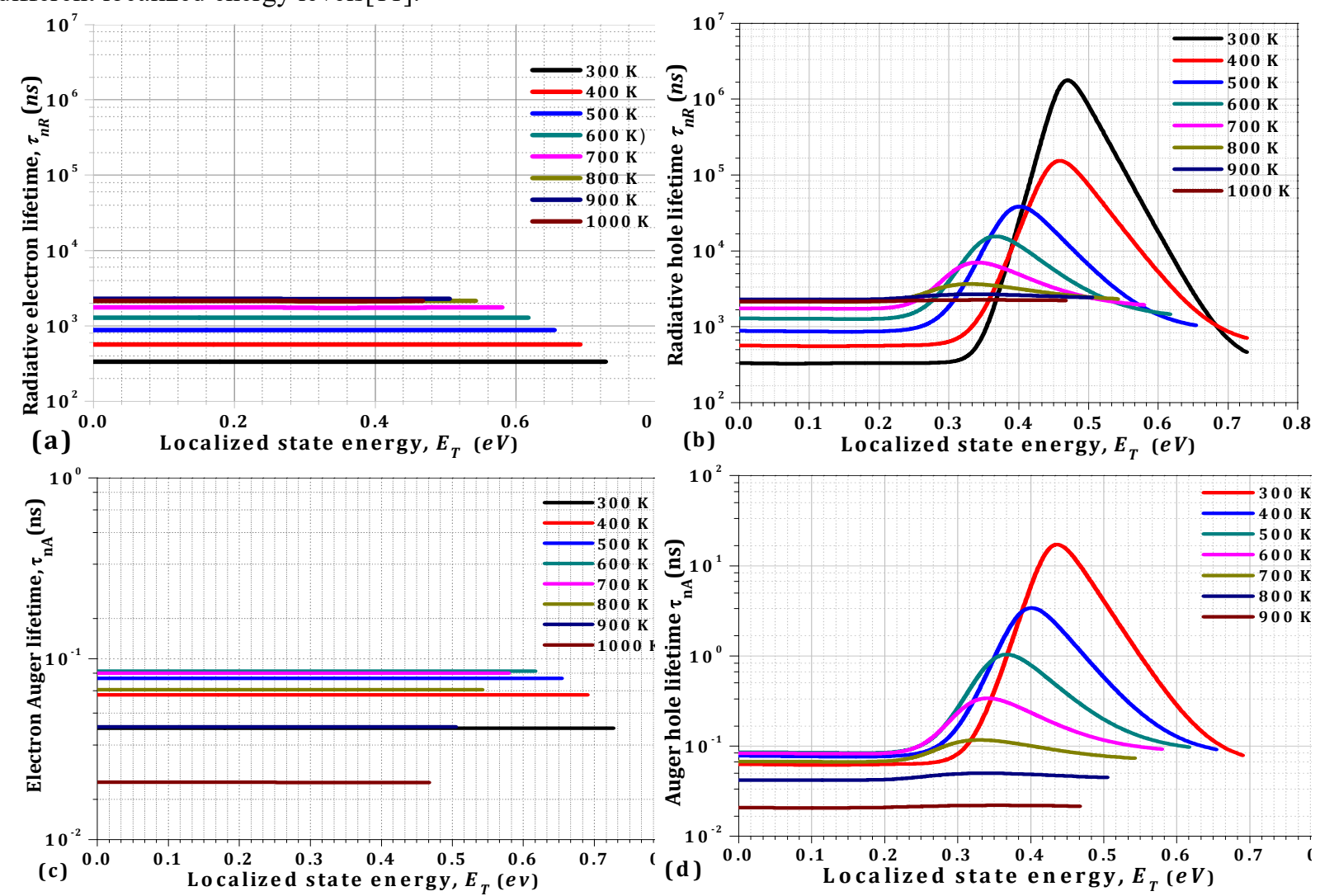

Figure 7:The lifetimes at different temperatures as a function of localized state energy, (a) the excess electrons\& (b) the excess holes' radiative lifetimes and (c) the excess electrons\& (d) the excess holes Auger lifetimes

\subsection{The temperature dependence Shockley-Read-Hall lifetime}

Figure 8illustrates the SRH lifetimes at different temperatures as a function of localized state energy for (a) excess electrons and (b) excess holes. The graphs are drawn using Eqns. 
$\tau_{n S R H}^{\prime}=\frac{\left.\tau_{n 0}\left(p_{0}+p_{1}+\delta p\right)\left(1+I_{n}\right)+\tau_{p 0}\left(n_{0}+n_{1}\right)\left(1+I_{n}\right)+\delta p\right)}{\left(p_{0}+n_{0}\left(1+I_{n}\right)+\delta p\right)\left(1+I_{n}\right)}$.

for Figure 8 (a) and

$$
\tau_{p S R H}^{\prime}=\frac{\delta p}{U_{S R H}^{\prime}}=\frac{\delta p \delta n}{\delta n U^{\prime} S R H}=\left(1+I_{n}\right) \tau_{n S R H}^{\prime}
$$

Figure 8 (b). As predicted earlier in Figure 5 and Figure 6, since the recombination and the trapping of carriers are negligible for localized levels near the valence and conduction band edges (acceptor and donor regions), the magnitude of both SRH lifetimes reaches a maximum value in these regions. The SRH lifetime in general decreases with increasing temperature, due to the dominance of trapping over emission at lower temperatures. This increases the SRH recombination rates and reduces the SRH carrier lifetimes at these centers, as shown in Figure 8 . The SRH lifetime for excess electrons has a minimum value at the sensitization states, as shown in Figure 8(a), and this point of minimum SRH lifetime shifts toward the conduction band edge with decreasing temperature.
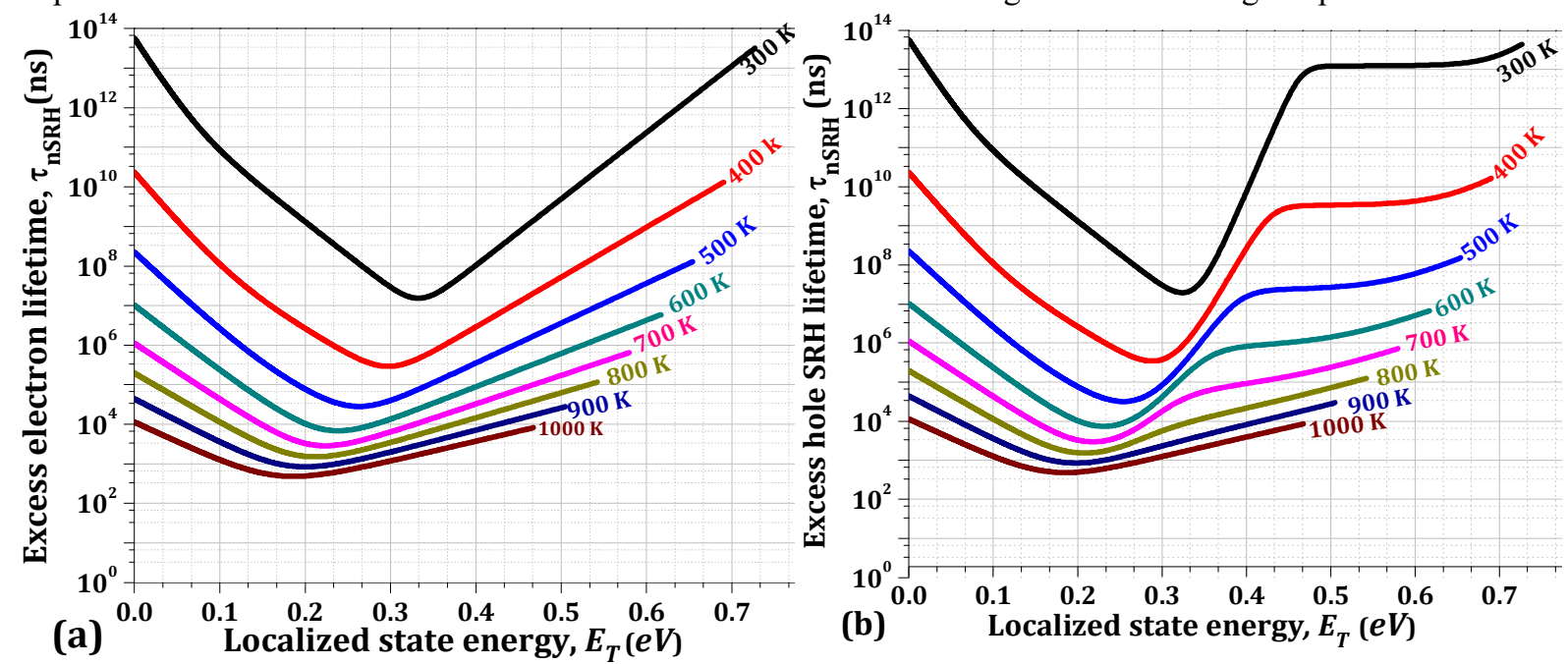

Figure 8:The SRH lifetime at different temperatures as a function of localized state energy (a) the excess electrons and (b) the excess holes.

The sensitization effect increases the SRH lifetime of excess holes in the region where the electron trap is influential (powerful). At very low temperatures, the SRH lifetime curves for holes become smooth in electron trap region and at high temperature slightly changes to the fashion of electron lifetime form smooth crescents at the bottom and symmetrically about the mid-gap. As temperature increases, the SRH lifetime for holes and electron decreases in the entire regions and localized state sensitization tends to increase the excess electron lifetime. The effect of minority carrier traps in the bulk of a semiconductor can be neglected at high temperatures. The ShockleyRead-Hall lifetimes in general decreases with increasing temperature as reported by [12]. The SRH carrier lifetimes small at recombination center, as shown in Figure 8. Therefore, the SRH carrier recombination lifetimes depends on the deep level density carrier concentrations[10].

\section{Conclusion}

The results obtained from the description of the effect of free carrier trap, the localized regions have different traps for their different localized energy levels. Depending on the interactions of localized states with the conduction or valence bands, the energy level dependence plot of the trap can be divided into five regions (i.e. acceptor, hole trap, recombination center, electron trap and donor regions). Most of the impurities are in the $n_{T}$ state for acceptor and in $p_{T}$ state for donor regions. In these regions (acceptor and donor) the effects of the traps of a localized center negligibly small. The energy value of this region is approximately equal or less than to thermal energy $k_{B} T$.

In hole trap region, the probability of emission of hole much greater than the probability of capture of electron i.e. $\sigma_{p} \gg \sigma_{n}$ ) and the process reverse for electron tap region but in the recombination center approximately the capture cross section $\sigma_{n}=\sigma_{p}$ which represents the probability of taps capturing carriers. From the energy level $0.08 \mathrm{eV}$ to $0.15 \mathrm{eV}$, hole trapping has maximum values and thus the hole trapping effect becomes dominant and for the energy level equal to $0.42 \mathrm{eV}$, the electron trapping has maximum values in this sample.

In general, we conclude that, the free carrier traps are relatively inactive at high temperatures compared to low temperatures. Only deep levels can trap free carriers at higher temperatures and trap is decreases with increasing temperature as function of localized state energies. All the radiative, Auger and SRH lifetimes are function of temperature and the Auger lifetime is dominated in the semiconductor gallium antimonide sample. 


\section{References}

1. Neamen, D.A., Semiconductor Physics and Devices:Basic Principles, 4th Edition 2003.

2. Kittel, C., Introduction to solid state Physics, 8th ed. . .

3. Kenichiro Tanaka*, H.U., Hidetoshi Ishida, Masahiro Ishida, and a.T. Ueda, Effects of hole traps on the temperature dependence of current collapsein a normally-OFF gate-injection transistor Japanese Journal of Applied Physics 55, 0541012016.

4. Li, S.S., Semiconductor Physical Electronics, . Springer Science+Business Media 2006.

5. Rupavatharam, V., "Modeling of QE, I-V Characteristics of MSM (Metal Semiconductor-Metal)," Mercuric Iodide Thin Films with MEDICITM 2004.

6. H. Schlangenotto, H.M.a.W.G., "Temperature Dependence of the radiative Recombination Coefficient in Silicon,. Physical Status of Solid, 1974. Vol. 21: p. pp. 357-367

7. F.Palumbo, S.P., F.Aguirre, R.Winter I.Krylov and M.Eizenberg, Temperature dependence of trapping effects in metal gates/Al2O3/InGaAs stacks Solid-State Electronics, vol. 35 (11), 12-18, 2017., 2017.

8. $\quad$ Dresselhaus, M.S., SOLID STATE PHYSICS: Optical Properties of Solids Vol. 2.

9. Ashcroft, N.W., Solid State Physics. 1976.

10. V. N. Abakumov, V.I.P., and I. N. Yassievich,, Nonradiative Recombinations in Semiconductors (NorthHolland, Amsterdam)1991.

11. Sermage, B., J.P. Heritage, and N. K. Dutta, Temperature dependence of carrier lifetime and Auger recombination in $1.3 \mu \mathrm{m}$ InGaAsP. Vol. 57. 1985. 5443-5449.

12. Schenk, A., A Model for the Field and Temperature Dependence of Shockley-Read-Hall Lifetimes in Silicon. Solid-State Electronics, vol. 35 (11), 1585-96, 1992., 1992. 